\title{
La introducción de las teorías raciales en la arqueología española: Manuel de Assas y Ereño ${ }^{1}$
}

\section{The introduction of racial theories in Spanish Archaeology: Manuel de Assas y Ereño}

\author{
Jordi VIDAL \\ Universitat Autònoma de Barcelona \\ Departament de Ciències de l'Antiguitat i de l'Edat Mitjana \\ Universitat Autònoma de Barcelona \\ 08193 Bellaterra (Cerdanyola del Vallès) \\ Jordi.Vidal.Palomino@uab.cat
}

Recibido: 30/01/2013

Aceptado: 08/03/2013

\begin{abstract}
RESUMEN
El objetivo del artículo es analizar las ideas raciales en la obra arqueológica de Manuel de Assas y Ereño. Concretamente estudiaremos el origen intelectual de sus ideas, la formulación de su pensamiento filoario y las posibles trazas de antisemitismo en sus trabajos sobre fenicios y púnicos, así como la recepción que tuvieron sus ideas raciales en España.
\end{abstract}

Palabras Clave: Racismo. Arianismo. Antisemitismo. Estudios indoeuropeos.

\section{Abstract}

The aim of this paper is to analyze racial ideas in the archaeological work of Manuel de Assas y Ereño. We specifically study the intellectual origins of his ideas, his Aryanism, the possible traces of anti-Semitism in his works on Phoenicians and Punics and the reception of his racial ideas in Spain.

KeY words: Racism. Aryanism. Anti-Semitism. Indo-European studies. 


\section{Introducción}

La figura pionera de Manuel de Assas y Ereño (Santander, 1813-1880) dentro de la arqueología y del orientalismo español ha sido justamente reivindicada en estos últimos tiempos gracias a los trabajos de autores como Víctor M. Renero Arribas (2004. Véase también Peiró Martín y Pasamar Alzuria 1996: 73 y 144s.; Romero Recio 2005 y 2006). En este proceso de recuperación, igualmente importante ha sido su inclusión en los principales diccionarios sobre historiadores y arqueólogos españoles publicados recientemente (Peiró Martín y Pasamar Alzuria 2002: 91s.; Mora 2009). En general, dichos trabajos han servido para rescatar del olvido a un personaje de relevancia en el desarrollo de las disciplinas antes citadas, y cuyo magisterio fue reconocido de forma explícita por figuras tan destacadas como las de José Ramón Mélida (1906: 9s.) y Francisco de Paula Álvarez-Ossorio (1934: 2).

Es precisamente gracias a los trabajos antes mentados que los principales datos biográficos de Assas resultan hoy fácilmente accesibles y contrastables. De ahí que a continuación únicamente recordaremos de forma sumaria algunos de esos datos, sobre todo aquellos relacionados con la arqueología y los estudios orientales.

A pesar de una primera licenciatura en derecho obtenida en las universidades de Valladolid y Central de Madrid (1836), sus principales intereses académicos fueron la lingüística oriental comparada y la arqueología. Su formación en estos campos se inició en 1846 en la Universidad Central, donde estudió árabe, hebreo y griego. Posteriormente, en 1852 llevó a cabo un viaje por Francia, Suiza, Alemania y Rusia ejerciendo como delegado de la Academia de Arqueología y Geografía del Príncipe Alfonso. Especial importancia tuvo su estancia en París, donde siguió cursos de sánscrito, celta, antigüedades egipcias y arqueología en la cátedra de Historia Antigua de la Sorbona. De esta forma entró en contacto directo con la academia francesa, cuya influencia se identifica rápidamente a lo largo de sus trabajos.

En su madurez obtuvo las cátedras de Sánscrito en la Universidad Central (1856-1858) y de Elementos de Arqueología en la Escuela Superior de Diplomática (1867-1880), además de ser miembro de la Real Academia de la Historia (1857) y de la Academia Arqueológica de Bélgica (1858).

En el ámbito del orientalismo, su principal mérito fue el de introducir en España unos estudios sobre los que no existía ninguna tradición hasta entonces (Peiró Martín y Pasamar Alzuria 1996: 144). Con todo, desde un punto de vista estrictamente académico, lo cierto es que no realizó nin- guna aportación relevante a la disciplina. En cambio, sus trabajos arqueológicos sí tuvieron mucha mayor trascendencia. Así, estudió y catalogó los monumentos megalíticos en España, cuyos resultados fueron publicados en el Semanario Pintoresco Español, revista que dirigió entre 1839 y 1857 . Todavía más importante fue su identificación del arte visigodo (que hasta entonces se creía inexistente) en una veintena de elementos arquitectónicos de la ciudad de Toledo (Assas 1848).

Sin embargo, si bien su biografía resulta fácilmente accesible, buena parte de la obra de Assas continúa siendo hoy en día poco conocida y analizada. En el presente artículo nos vamos a centrar en un aspecto concreto de esa obra, esto es, en la importancia que Assas concedía a la cuestión racial como elemento determinante en el desarrollo histórico de las sociedades antiguas.

Las ideas raciales de Assas se basaban fundamentalmente en la tradición intelectual francesa, algo lógico si tenemos en cuenta su periodo de formación en París. Dichas ideas se hallan bien sistematizadas en su Crónica de la provincia de Santander (Assas 1867), donde el autor nos ofrece su propio ensayo sobre prehistoria europea. Allí realiza una división tripartita de las razas humanas (blanca/caucásica, amarilla/mongólica y negra/etiópica), tomada directamente del influyente trabajo de Arthur de Gobineau, Essai sur l'inegalité des races humaines (Paris 1853-1855). Gobineau fue uno de los más influyentes teóricos del racismo europeo surgido de la combinación del cientifismo reduccionista de base biológica y la metafísica romántica de la raza (Bernal 1993 [1987]: 316ss.; véase también Buenzod 1967; Biddiss 1970; Poliakov 1987 [1971] 245ss.; Banton 1998: 62ss.). De acuerdo con su clasificación racial, la raza blanca se dividiría a su vez en dos ramas. La primera era la rama aria, originaria de la India asiática y a la cual pertenecerían todas las poblaciones europeas actuales. La segunda era la rama arameo-caucásica, donde se situaban todas las poblaciones semíticas conocidas (hebreos, árabes, fenicios, etc.) así como los habitantes de casi todas las islas mediterráneas (Assas 1867: 15).

Esta división racial condicionó decisivamente la visión de Assas acerca de la prehistoria y protohistoria de la Península Ibérica, sirviéndole como marco interpretativo a partir del cual formular sus ideas decididamente filoarias. En los dos apartados siguientes repasaremos en primera instancia la importancia fundamental que según Assas tuvo la raza aria en el desarrollo de la civilización occidental. En segundo lugar analizaremos la imagen negativa que ofreció Assas de fenicios y cartagineses. El objetivo de ese apartado será el de determinar si Assas, de acuerdo con el clima intelectual de la 
época en Europa, además de su explícita postura filoaria desarrolló un pensamiento antisemita basado en prejuicios raciales, o bien se limitó a recoger una serie de tópicos antifenicios de larga tradición en la historiografía española.

\section{Teorías filoarias y el proceso civilizador de la Península Ibérica}

Tal y como señala Renero Arribas en sus apuntes biográficos sobre Assas, 1852 fue un año clave para su formación intelectual, en especial su estancia en París y sus clases de lenguas indoeuropeas (sobre todo de sánscrito) con Théodore Pavie (Renero Arribas 2004: 96). Allí adoptó definitivamente algunos de los que después se convertirían en los ejes principales de su pensamiento: la fascinación por el sánscrito y la lingüística indoeuropea, la identificación entre raza y lengua, la aplicación de la taxonomía racial a los estudios históricos, una actitud decididamente filoaria y la aceptación de la cuestión racial como principio explicativo fundamental de la historia humana.

De nuevo en Madrid Assas trató de difundir los estudios de lingüística indoeuropea mediante la impartición de cursos sobre la materia en el Ateneo de Madrid y en la Universidad Central. Al mismo tiempo trabajó a nivel político para la creación de una cátedra de Lengua Sánscrita, tal y como se aprecia en la siguiente carta del 2 de junio de 1856 dirigida a Francisco de Luján Miguel Romero, que por aquel entonces se hallaba al frente del Ministerio de Fomento: ${ }^{2}$

\section{Exmo Sr.}

D. Manuel de Assas caballero de la Real y distinguida órden española de Carlos III, abogado, académico de la Arqueología española, á V.E. con el debido respeto expone; que, son bien conocidos, y sobre todo de V.E., los estudios hechos recientemente en varios puntos de Europa, principalmente en Alemania, sobre la comparación de las lenguas orientales con las occidentales de Europa, especialmente del sanscrito, lengua modelo, producto de la sabiduría de la India, y cuya filiación el célebre Bopp ${ }^{3}$ y otros eminentes filólogos han descubierto en el griego y celta antiguos, en las lenguas germánicas, y de la consiguiente en casi todas las lenguas y dialectos de la actual Europa civilizada. Es ya pues, materia incuestionable en etnografía, que las principales naciones de la Europa culta forman en la humanidad una sola rama conocida por los autores bajo el nombre de indo-germánica. Sería ofender la ilustración de V.E. entrar en largas consideraciones para demostrar cuan interesada está España en la introducción y prosecución de esta clase de estudios; pues invadida primero por los celtas, después por los romanos y godos, y establecidos en muchos puntos de sus costas los griegos; los rastros de estos pueblos que hablaban lenguas afines, han quedado impresos en la lengua castellana y en los nombres de montes, ríos y lugares. Sin duda por la dificultad, por lo ingrato de la tarea, y por la ninguna recompensa que el público ó el gobierno han ofrecido hasta ahora á esta clase de estudios, ningún español ha habido que se haya dedicado á ellos con noticia del público hasta que el exponente, fiado más en su entusiasmo por la ciencia que en sus escasos conocimientos, $\mathrm{y}$ tendiendo más bien a llamar la atención del público y del gobierno a esta clase de estudios, comenzó un curso de lecciones sobre lengua sanscrita en el Ateneo de esta corte en los dos años de 1853 á 55. Mas, como V.E. sabe, no es este pequeño esfuerzo individual lo que puede satisfacer á la España ilustrada que entra actualmente por todos los caminos en la vía de la civilización dirigida por las ciencias, ni a un gobierno celoso de no ver á su nación retrasada en la marcha de los conocimientos humanos. Mucho influye en la frecuencia y armonía de las relaciones internacionales mostrar descendientes de un mismo tronco y expresando sus sentimientos é idéas en variaciones de una misma lengua a pueblos creídos hasta el día de opuesta procedencia y de antipáticos idiomas, y no influye menos en la unión á que aspiran, en la fusión á que tienden tan rápidamente por las grandes invenciones del vapor y del telégrafo, las diferentes y antes rivales razas habitadoras de la Península, encontrar que los celtas de Portugal, de Galicia y de Cantabria, los griegos y provenzales de Cataluña y Valencia, y las razas germánico-latinas de todo el norte y centro de España, proceden de un tronco común, y sus diferentes dialectos son hijos de una misma madre, que es la lengua sanscrita de la India. El exponente, no solo ha dedicado mucho tiempo en París al estudio de esta lengua de los pueblos indo-germánicos con el distinguido profesor del Collège de France Mr. Théodore Pavie, ${ }^{4}$ sino que para poder sacar mayor fruto de este estudio, ha completado el otro que de muchos años llevaba preparado ya, sobre las lenguas semíticas, el hebreo y el árabe, que según los últimos descubrimientos etnográficos son la base de todos los dialectos hablados en el África septentrional, excepto el Egipto; es decir, en la región que por siete siglos dió á España pobladores y dominadores; lo cual se aviene perfectamente con las relaciones de la Historia, pues los fenicios colonizadores del Africa septentrional hablaban el hebreo, cuyos rastros han quedado en los actuales montañeses del Atlas, siendo los habitantes de los valles y costas 
hijos de los árabes, últimos conquistadores y actuales poseedores de esta tierra. En vista de esto el exponente espera de la ilustración de V.E. y de su ardor por la propagación de las ciencias, que con tanto fruto cultiva, que hará señalar su administración con la introducción en España de una enseñanza de la lengua sanscrita comparada con las demás indo-germánicas (céltica, germánicas, griega, latina y sus dialectos modernos), y con las semíticas árabe y hebrea. Por todo lo cual,

A V.E. suplica que autorice la explicación de una enseñanza de esta clase en la Universidad central para el próximo curso de 56 á 57, incluyéndola en el plan de estudios, sin que sea obstáculo para su fundación el esperar la dotación de las Cortes; pues así como voluntaria y patrióticamente ha explicado ya el exponente por espacio de dos años en el Ateneo, se halla dispuesto a cumplir la orden satisfactoria en que V.E. le encomendase esta enseñanza.

Dios guie á V.E. ms. as. Madrid 2 de Junio de 1856. Manuel de Assas.

(AGA, legajo 114-17, caja 31/15303)

Como se observa en la carta, Assas defendía con la vehemencia propia de la época la necesidad de introducir los estudios de lingüística indoeuropea en la universidad española mediante la creación de una cátedra de lengua sánscrita en la Universidad Central de Madrid. De esta forma, España seguiría los pasos de países como Francia, Inglaterra o Alemania, donde ya a principios del siglo XIX se habían creado las primeras cátedras de lengua sánscrita (Bernal 1993 [1987]: 220). Es interesante observar cómo, al margen de los argumentos estrictamente académicos, Assas utilizó en su carta al ministro también dos argumentos de tipo político para tratar de convencerle definitivamente acerca de la conveniencia de crear dicha cátedra: (1) la necesidad de equiparar a España con el resto de países de la Europa Occidental, donde, tal y como hemos comentado, dichas cátedras ya existían; (2) el hecho de que los estudios indoeuropeos, al demostrar la existencia de una primitiva cohesión lingüística, podían contribuir de forma decisiva a mantener y reforzar la unidad de los pueblos de España. Las palabras de Assas cobran pleno sentido si tenemos en cuenta que precisamente durante la primera mitad del XIX surgieron movimientos como el catalanismo, que podían percibirse desde determinados sectores como un cuestionamiento de la unidad de España. Finalmente, Assas tuvo (relativo) éxito en su empeño, logrando la creación de la cátedra de sánscrito en la Universidad Central. Con todo, cabe señalar que dicha cátedra carecía de dotación económica, lo que obligó a Assas a ejercer la docencia de forma gratuita, tal y como consta explícitamente en su Hoja de Servicios:
1856 á 1858 - Explicó gratuitamente en la Universidad central, la Lengua Sanscrita, autorizado al efecto por Reales Ordenes de 27 de Junio de 1856, y de 16 de Octubre de 1857, durante dos cursos académicos.

(Archivo-BNE, BN 3119/020)

Por lo que se refiere a su obra científica, el enorme interés de Assas por los estudios relacionados con las lenguas indoeuropeas y la raza aria condicionó de forma decisiva su visión sobre la prehistoria y la historia antigua de Europa. En este sentido, resultan fundamentales las primeras páginas de $\mathrm{su}$ ya mentada Crónica de la provincia de Santander, donde recogía de forma generosa las teorías histórico-lingüísticas de Friedrich Schlegel, uno de los fundadores de los estudios indoeuropeos (Villar 1996: 18ss.).

Assas demostró un especial interés en la cultura celta, un término, el de celta, que a menudo utilizaba simplemente como sinónimo de ario, mostrando en cierta forma la evidente dificultad existente a la hora de definir ese concepto (Ruiz Zapatero 1993). Su evidente arianismo le llevó a aceptar de forma acrítica diversos tópicos acerca de la superioridad racial aria, llegando a afirmar que "los salvajes se puede decir que no tienen historia sino desde la época en que les han visitado los europeos" (Casado Rigalt 2006: 33). De los celtas/arios admiraba su legendaria belicosidad (Assas 1867: 42), así como su superior capacidad intelectual, lo que les permitía alcanzar los más altos logros civilizadores.

En este sentido resulta especialmente significativa su expresiva descripción del estado de la Península Ibérica antes de la llegada de las poblaciones de origen celta:

sus habitantes eran sencillos en los vestidos $\mathrm{y}$ armas, y frugales en las comidas (...) ignoraban las ciencias; y el comercio, si le tenían, debía estar limitado á las costas (...) Debiendo hallarse las artes tan atrasadas como todo lo demás relativo a la civilización, y no habiendo llegado a nosotros ni noticia de monumento alguno de aquella primitiva época, podemos suponer que los españoles habitarían a la sazón en las cavernas de las rocas, o en los huecos de los troncos de árboles carcomidos, o bien en chozas pajizas o cabañas formadas con ramas o troncos de árboles; o finalmente en cualquiera de las demás clases de albergues en que moran hoy diversas naciones no civilizadas.

(Assas 1857a: 130)

Únicamente la llegada de los celtas, a los que califica como "la vanguardia de la raza blanca" 
(Assas 1867: 42), a través de los Pirineos permitió el surgimiento de la civilización en la Península, con el consiguiente abandono del salvajismo propio de las poblaciones prearias. Para Assas, por lo tanto, las más importantes virtudes y los más altos logros de la historia y de la civilización eran elementos que estaban inevitablemente ligados a la superior capacidad física, intelectual y emocional de los pueblos de raza aria.

Con estos planteamientos Assas recogía directamente uno de los grandes temas del racismo fisiológico europeo, según el cual tan solo la raza aria era capaz de crear cultura, frente a la esterilidad y el parasitismo que caracterizaría al resto de razas, en especial a los semitas. Dicha idea, enunciada a mediados del siglo XIX por autores como el orientalista Ernest Renan o el propio Gobineau (Bernal 1993 [1987]: 317ss.), fue especialmente celebrada por el nazismo, llegándola a reproducir el propio Hitler en Mein Kampf (Chapoutot 2008: 32).

\section{3. ¿Antisemitismo? Fenicios y púnicos en la Península Ibérica}

Rápidamente el arianismo filológico-racial se asoció con las crecientes tendencias antisemitas que se estaban desarrollando en la Europa del siglo XIX, dando lugar a "una nueva formulación en la dicotomía racial ario-semita" (Álvarez Chillida 2002: 220). Esa dicotomía con frecuencia se planteó en términos históricos como una especie de interminable combate a muerte entre arios y semitas, donde estos últimos estarían empeñados en conspirar de forma más o menos explícita para la destrucción de la superior civilización europea aria y la imposición de su linaje sobre el resto del mundo. Ya en el siglo XX, la ideología völkisch explotó a fondo y amplificó esa idea de la lucha a muerte entre arios y semitas con los resultados dramáticos que todos conocemos. En el ámbito de la historia antigua la oposición arios-semitas tuvo también un importante desarrollo en la historiografía antisemita europea. Un buen ejemplo de ello lo ofrece el historiador francés Jules Michelet, uno de los más claros exponentes del antisemitismo del siglo XIX. Así, en su obra Histoire Romaine Michelet no dudaba en definir a fenicios y cartagineses como un pueblo avaro, capaz de perpetrar las más horribles crueldades en el transcurso de sus prácticas religiosas $\mathrm{y}$, por supuesto, mucho más amante del vil comercio que de la guerra heroica. En este sentido, resulta especialmente significativa la oposición radical que describía entre romanos (arios) y cartagineses (semitas) a propósito de las guerras púnicas, una oposición que en ningún caso era circunstancial sino que ejemplificaba la eterna lucha entre las razas aria y semita:

Ce n'est point sans raison que le souvenir des guerres puniques est resté si populaire et si vif dans la mémoire des hommes. Cette lutte ne devait pas seulement décider du sort de deux villes ou de deux empires; il s'agissait de savoir à laquelle des deux races, indo-germanique ou sémitique, appartiendrait la domination du monde (...) D'un côté, le génie héroïque, celui de l'art et de la législation; de l'autre, l'esprit d'industrie, de navigation, de commerce. Ces deux races ennemies se sont partout rencontrées, partout attaquées. Dans la primitive histoire de la Perse et de la Chaldée, les héros combattent sans cesse leurs industrieux et perfides voisins. Ceux-ci sont artisans, forgerons, mineurs, enchanteurs. Ils aiment l'or, la sang, le plaisir. Ils élèvent des tours d'une ambition titanique, des jardins aériens, des palais magiques, que l'épée des guerriers dissipe et efface de la terre.

(Michelet 1833 [1830]: 245s.)

En dicho pasaje se aprecian con claridad algunos de los principales tópicos antisemitas que poblaron la literatura europea del XIX: la avaricia, la crueldad, su pasión por el comercio, su ineptitud para la guerra y su insoportable falta de heroísmo consecuencia inevitable de su naturaleza débil y afeminada.

Dicho esto, al repasar la producción bibliográfica de Assas relacionada con la arqueología vemos cómo muchos de estos tópicos característicos del antisemitismo racial europeo también aparecen en sus obras.

Así, al describir el origen asiático de los pueblos semitas Assas ya hacía referencia a su inferioridad militar respecto a sus poderosos vecinos griegos y egipcios, y a su recurso al comercio como medio poco honorable a través del cual superar su lamentable incapacidad bélica:

Los individuos de esta raza (...) atravesaron el monte Zagros, y se extendieron por fin hasta las riberas del Tigris, del Éufrates y del Indo. El rey Stabrobates los contuvo junto a este célebre rio, y los griegos por el Norte y los egipcios por el Mediodía les fueron insuperables obstáculos en sus incesantes invasiones hacia el Poniente. Pero entre la Grecia y el Egipto quedaba abierto el camino del mar Mediterráneo; y por medio de este, operó, en parte, el comercio lo que la guerra no pudo realizar: por allí vinieron al Occidente los fenicios.

(Assas 1867: 18) 
Centrándose de manera específica en el caso fenicio, Assas destacaba de forma vehemente su legendaria avaricia y crueldad así como, de nuevo, su malsano amor por el comercio:

Eran los fenicios avaros y crueles, y envidiosos enemigos de las empresas mercantiles de otros pueblos (...). Añadiendo a tan malas propiedades un sin número de vejaciones ejercidas sobre las regiones por ellos colonizadas, se habían atraído el odio universal.

(Assas 1857b: 252)

Finalmente, Assas, ofreciendo una relectura particular de una noticia recogida por Justino y ampliamente reproducida por la historiografía española desde época medieval (Ferrer Albelda 1996: 23), definía una antigua y natural oposición entre españoles y fenicios, al tiempo que insistía en la cobardía e ineptitud militar de los últimos:

Los españoles, que ya los detestaban, sintiéndose agraviados en una contienda causada por la avaricia de aquellos mercaderes, tomaron las armas; y después de apoderarse de varios establecimientos fenicios, marcharon á atacar á Cádiz. Los de Fenicia, llenos de temor, como poco acostumbrados á la guerra, pidieron auxilio a los cartagineses (...).

(Assas 1857b: 252)

En este sentido, resulta muy ilustrativo comparar el pasaje anterior con la noticia del propio Justino a la que antes hacíamos referencia, donde se aprecia claramente la voluntad de Assas de envilecer la imagen de los fenicios aún a costa de manipular las fuentes:

En efecto, cuando los gaditanos recibieron en sueños la orden de trasladar a Hispania el culto de Hércules desde Tiro, de donde también procedían los cartagineses, y fundaron allí una ciudad, puesto que los pueblos vecinos de Hispania, que veían con malos ojos el engrandecimiento de la nueva ciudad, hostigaban a los gaditanos con la guerra, los cartagineses enviaron ayuda a sus hermanos de raza. Allí, en una expedición victoriosa liberaron a los gaditanos de la injusticia y con una injusticia mayor aún unieron una parte de la provincia a su dominio.

(Justino, Epitome, XLIV, 5, 2-3. Trad. J. Castro Sánchez 1995)

Las diferencias entre la cita de Justino y el párrafo de Assas son evidentes y muy significativas. Así, en primera instancia, mientras que Justino entendía el ataque de los indígenas contra Gadir como un acto esencialmente injusto, Assas lo interpretaba como una defensa legítima y necesaria contra la avaricia insaciable de los fenicios. Por otra parte, mientras que Justino consideraba que la ayuda prestada por los cartagineses a los habitantes de Gadir era del todo lógica teniendo en cuenta su común origen étnico, sin realizar ningún juicio de valor posterior, Assas aprovechaba esa circunstancia para destacar nuevamente la conocida cobardía fenicia que les llevó rápidamente a solicitar la ayuda cartaginesa ante la amenaza indígena. Por lo demás, conviene recordar que tanto la cronología como, incluso, la historicidad misma de esa información transmitida por Justino han sido objeto de muy diversas interpretaciones (Ferrer Albelda 1996: 57; Domínguez Monedero 2007: 215).

Sin embargo, considero que ese conjunto de referencias analizadas en realidad están más relacionadas con una secular tradición historiográfica española claramente antipúnica que con el antisemitismo racial europeo del siglo XIX. En este sentido Eduardo Ferrer Albelda ha mostrado cómo mucho antes del surgimiento del antisemitismo de origen racista autores como Florián de Ocampo (1513-1590?), Juan de Mariana (1536-1624), José Morales Santiesteban (1799-?) o Modesto Lafuente (1806-1866) ya difundieron una imagen esencialmente negativa del mundo fenicio y, sobre todo, púnico, basada en los prejuicios recogidos en las fuentes grecolatinas (Ferrer Albelda 1996: 33, 41, 69ss.; véase también García Fernández y Fernández Götz 2010: 55s.). Así, en la Crónica general de España de Ocampo (1548) los púnicos ya eran descritos como un pueblo avaro, impío, pérfido y cruel, una imagen que reaparece incluso amplificada en la Historiae de rebus Hispaniae (1592) de Mariana y, por supuesto, en la historiografía romántica del XIX, tal y como se aprecia en la siguiente cita de Lafuente: "Por eso, y a pesar de las repugnantes condiciones de su carácter, y aun cuando siguió en sus procederes caminos poco honrosos, prestó indudables servicios al progreso universal" (Wulff 2003: 144). Sin variaciones significativas ni elaboraciones intelectuales distintas, todos estos tópicos son los que reaparecen en los textos de Assas sobre la presencia de fenicios y púnicos en Hispania.

Otro elemento que nos lleva a vincular los planteamientos antipúnicos expuestos por Assas con esa antigua tradición historiográfica española antes que con el antisemitismo racial europeo del siglo XIX es el hecho de que en su correspondencia, donde como hemos visto se refiere a la necesidad de potenciar el estudio de las lenguas hebrea y árabe, jamás se deslicen comentarios de corte 
antisemita ni referencias de ninguna clase a los principales teóricos del antisemitismo.

\section{Consideraciones finales}

El pensamiento de Assas partía de la aceptación de una serie de principios determinantes como eran la identificación entre lengua y raza, la superioridad racial aria, la importancia de la raza como motor de la historia, etc. Dichos principios condicionaron de forma absoluta su particular visión de la prehistoria y la historia antigua de Hispania, donde las poblaciones de origen indoeuropeo (celtas) fueron las responsables directas del influjo civilizador que sacó a los primeros habitantes de la Península Ibérica del salvajismo en el que se hallaban inmersos. Está claro que Assas de esta forma recogía las ideas racistas que por entonces se desarrollaban en Europa. Con todo, conviene recordar aquí que no todos los investigadores relacionados con los estudios indoeuropeos abrazaron las ideas de la superioridad racial aria y la identificación entre raza y lengua. Un ejemplo paradigmático en este sentido es el de Max Müller (18231900), eminente orientalista alemán y profesor de Filología comparada en la Universidad de Oxford, quien siempre rechazó de plano otorgar cualquier connotación racial a sus estudios sobre lingüística indoeuropea (Figueira 2002: 44s.). El ejemplo de Max Müller enseña que el camino racista tomado por Assas no era un camino inevitable.

Más difícil resulta valorar la difusión y el impacto que tuvieron las teorías de Assas en los estudios sobre la prehistoria y la historia antigua en la España del siglo XIX. Lo que sí está claro es que Assas dispuso de canales diversos y privilegiados a partir de los cuales difundir sus ideas. Así, al margen de sus artículos y libros y de sus clases en las cátedras de sánscrito y arqueología, Assas pronunció en el Ateneo de Madrid diversos ciclos de conferencias directamente relacionados con las materias que aquí hemos analizado y donde a buen seguro dio a conocer sus ideas acerca de la importancia de la raza en los estudios de prehistoria y protohistoria. Esta es la información que consta en su Hoja de Servicios:

1846 á 1849 - Historia de la Arquitectura Española, en el Ateneo de Madrid, durante tres cursos.

1849 á 1852 - Historia Universal de la Arquitectura, en dicho Ateneo de Madrid durante tres cursos.

1853 á 1854 - Lengua y Literatura Sanscrita, en el mismo Ateneo durante un curso.
1854 á 1856 - Arqueología Española, en el Ateneo, durante dos cursos.

1856 á 1858 - Lengua Céltica en el Ateneo, durante dos cursos.

1858 á 1860 - Arqueología Española, en el Ateneo, otros dos cursos.

(Archivo-BNE, BN 3119/020)

Dichos cursos indican que las ideas de Assas debían de ser bien conocidas entre los sectores cultos de la sociedad madrileña de mediados del XIX.

Desde un punto de vista estrictamente académico, sabemos que Assas concedía en sus clases de arqueología en la Escuela Superior de Diplomática especial atención a la cuestión racial. Así se aprecia, por ejemplo, en su Programa de la asignatura de arqueología, donde la lección 8 estaba dedicada a este tema, con los siguientes contenidos:

Las principales razas humanas y algunas de sus más importantes emigraciones. Progresos de cada una en la civilización, y su consiguiente importancia en la Arqueología.

$($ Assas 1876: 4)

En este sentido resulta igualmente significativo comparar brevemente los trabajos de Assas con los de su discípulo más reconocido, José Ramón Mélida. Ciertamente, ambos discrepan de forma sustancial en muchos aspectos centrales, como puede ser el hecho de que Mélida disminuyera considerablemente el papel histórico-civilizador atribuido por Assas a los celtas en favor de las colonizaciones mediterráneas. Sin embargo, ambos coinciden en la importancia otorgada al elemento racial ario, tal y como se aprecia en los siguientes fragmentos extraídos de dos de las obras de Mélida: "A la inteligente raza aria pertenecían los pobladores de la Grecia (...) La raza aria (...) rompe con todas las tradiciones hieráticas y espirituales del Oriente, y, emancipada, se entrega a la libre interpretación de la Naturaleza" (Mélida 1897: 7ss.); "Los griegos, raza privilegiada (...)" (Mélida 1896: 57). Es del todo plausible suponer que detrás de esas palabras de alguna forma se encuentran todavía presentes las antiguas enseñanzas de Assas, transmitidas desde su cátedra de arqueología en la Escuela Superior de Diplomática a alumnos como el propio Mélida, que estudió en dicha institución entre junio de 1873 y diciembre de 1875 (Casado Rigalt 2006: 32).

Por todo ello parece razonable concluir que Assas logró difundir con relativo éxito su particular visión de la prehistoria y la historia antigua universal y de España, fuertemente condicionada por la importancia decisiva concedida al elemento racial y la aceptación de la superioridad aria. 


\section{Notas}

1. Artículo producido en el marco del proyecto de investigación HAR2011-23572. Agradezco a Jordi Cortadella la lectura que hizo del artículo. Por supuesto, los errores son responsabilidad únicamente mía. Abreviaturas utilizadas: $\mathrm{AGA}=$ Archivo General de la Administración (Alcalá de Henares); Archivo-BNE = Archivo de la Biblioteca Nacional.

2. Si bien la carta no especifica el nombre del destinatario, la posterior respuesta, también conservada en el expediente de Assas en el AGA, confirma que la misma se dirigió al ministro de Fomento, cargo por aquel entonces ostentado por Francisco de Luján Miguel Romero (Urquijo Goitia 2008: 410).

3. Franz Bopp (1791-1867). Lingüista alemán. Fue profesor de Filología Oriental en la Universidad de Berlín y uno de los primeros investigadores responsables de sistematizar las similitudes entre las distintas lenguas de origen indoeuropeo. Entre sus obras destacan Vergleichende Grammatik des Sanskrit, Zend, Griechischen, Leteinischen, Litthauischen, Gothischen und Deutschen (Berlin 1833-1842) y Kritische Grammatik der SanskritaSprache in kürzerer Fassung (Berlin 1934) (Sternemann 1984).

4. Théodore Pavie (1811-1896). Orientalista francés. Fue profesor de sánscrito en el College de France durante el periodo 1852-1857. Entre sus obras más importantes relacionadas con el sánscrito destacan Fragments du Mahabharata (Paris 1844) y Bhojaprabandha (Paris 1855) (Despland 1996: 516).

5. Agradezco muy sinceramente a Mirella Romero Recio que me facilitara una copia de dicho programa.

\section{ReFERENCIAS Bibliográficas}

Almagro-Gorbea, M.; Ruiz Zapatero, G. (eds.) (1993): Los Celtas:Hispania y Europa. Actas, Madrid.

Álvarez Chillida, G. (2002): El Antisemitismo en España. La imagen del judio (1812- 2002). Marcial Pons, Madrid.

Álvarez-Ossorio, F. (1934): Notas biográficas del Exmo. Sr. D. José Ramón Mélida y Alinari. Anuario del Cuerpo Facultativo de Archiveros, Bibliotecarios y Arqueólogos, 1: 1-48.

AsSAS, M. DE (1857a): Nociones fisionómico-históricas de la arquitectura en España. Monumentos célticos. Semanario Pintoresco Español, XXII: 129-133, 140-141, 148-149, 155-158, 163-165 y 172-173.

Assas, M. DE (1857b): Nociones fisionómico-históricas de la arquitectura en España. Monumentos cartagineses. Semanario Pintoresco Español, XXII: 252-253.

Assas, M. DE (1867): Crónica de la provincia de Santander. Rubio y Compañía, Madrid.

Assas, M. DE (1876): Programa de la asignatura de arqueología. Imprenta de T. Fortanet, Madrid.

Banton, M. (1998): Racial Theories (Second Edition). Cambridge University Press Cambridge.

Beltrán, J.; Cacciotti, B.; Palma, B. (eds.) (2006): Arqueología, coleccionismo y antigüedad: España e Italia en el siglo XIX. Universidad de Sevilla, Sevilla.

Bernal, M. (1993) [1987]: Atenea negra. Las raíces afroasiáticas de la civilización clásica. Crítica, Barcelona.

BidDiss, M. D. (1970): Father of Racist Ideology: The Social and Political Thought of Count Gobineau. Weybright and Talley, London.

BuEnzod, J. (1967): La Formation de la pensée de Gobineau et l'Essai sur l'inégalité des races humaines. Nizet, Paris. Casado Rigalt, D. (2006): José Ramón Mélida y la arqueología española. Real Academia de la Historia, Madrid.

Castro SÁnchez, J. (1995): Marco Juniano Justino. Epitome de las Historias Filípicas de Pompeyo Trogo. Pompeyo Trogo. Prólogos. Fragmentos. Gredos, Madrid.

Снарочтот, J. (2008): Le national-socialisme et l'Antiquité. Presses universitaires de France, Paris.

Despland, M. (1996): Pavie, Théodore. En Laplanche 1996: 516.

Díaz-Andreu, M.; Mora Rodríguez, G.; Cortadella Morral, J. (eds.) (2009): Diccionario histórico de la arqueología en España. Marcial Pons, Madrid.

Domínguez Monedero, A. (2007): “Los fenicios en Occidente”. En Sánchez-Moreno 2007: 75-225.

FERRER Albelda, E. (1996): La España cartaginesa. Claves historiográficas para la historia de España. Universidad de Sevilla, Sevilla.

Figueira, D. M. (2002): Aryans, Jews, Brahmins: Theorizing Authority Through Myths of Identity. State University of New York Press, New York. 
García Fernández, F. J.; Fernández Götz, M. A. (2010): Esencialismo, normativismo, postmodernismo: las interpretaciones sobre la etnicidad en la Arqueología Española”, Gerión, 28: 53-84.

Gobineau, A. (1853-1855): Essai sur l'inegalité des races humaines. Librairie de Firmin Didot frères, Paris.

Laplanche, F. (ed.) (1996): Les sciences religieuses. Dictionnaire du monde religieux dans la France contemporaine. Beauchesne, Paris.

MélidA, J. R. (1896): Conferencias en el Museo Arqueológico Nacional, dadas en los días 24 de mayo, 7 y 14 de junio de 1896. Revista de Archivos, Bibliotecas y Museos, 4: 55-59.

MÉLIDA, J. R. (1897): Historia del arte griego. La España Editorial, Madrid.

MÉLIDA, J. R. (1906): Iberia arqueológica ante-romana. Est. Tip. de la viuda e hijos de Tello, Madrid.

Michelet, J. (1833): Histoire romaine. Première partie: République. Librairie classique et élémentaire de L. Hachette, Paris.

Mora, G. (2009): Assas y Ereño, Manuel. En M. Díaz-Andreu; G. Mora Rodríguez ; J. Cortadella Morral (eds.) 2007: 101-102.

Peiró Martín, I.; Pasamar Alzuria, G. (1996): La Escuela Superior de Diplomática (Los archiveros en la Historiografía Española Contemporánea). ANABAD, Madrid.

Peiró Martín, I.; Pasamar Alzuria, G. (2002): Diccionaro Akal de historiadores españoles contemporáneos (1840-1980). Akal, Madrid.

Poliakov, L. (1987) [1971]: Le mythe aryen. Essai sur les sources du racisme et des nationalismes. Complexe, Paris.

Renero Arribas, V. M. (2004): Manuel de Assas y Ereño. Zona Arqueológica, 3: 95-101.

Romero Recio, M. (2005): La Biblioteca de la Escuela Superior de Diplomática: la presencia de la Historia Antigua en la enseñanza española del siglo XIX a través de sus fondos. Gerión, 23: 345-370.

Romero Recio, M. (2006): La arqueología en la enseñanza española durante el siglo XIX: nuevas aportaciones a la luz de documentos inéditos. En J. Beltrán, B. Cacciotti y B. Palma (eds.) 2006: 581-601.

Ruiz Zapatero, G. (1993): El concepto de Celtas en la Prehistoria europea y española. En M. Almagro-Gorbea y G. Ruiz Zapatero (eds.) 1993: 23-62.

SÁnchez-Moreno, E. (ed.) (2007): Protohistoria y Antigüedad de la Península Ibérica. Vol. I: Las fuentes y la Iberia colonial. Sílex, Madrid.

Sternemann, R. (1984): Franz Bopp und die vergleichende indoeuropäische Sprachwissenschaft. Institut für Sprachwissenschaft der Universität Innsbruck, Innsbruck.

Urquijo Goitia, J. R. (2008): Gobiernos y ministros españoles en la Edad Contemporánea. Consejo Superior de Investigaciones Científicas, Madrid.

VILlar, F. (1996): Los indoeuropeos y los orígenes de Europa. Lenguaje e Historia. Gredos, Madrid.

WulfF, F. (2003): Las esencias patrias. Historiografia e historia antigua en la construcción de la identidad española. Crítica, Barcelona. 